\title{
A Fuzzy Qualitative Simulation Study of College Student's Mental Health Status
}

\author{
Xiying Deng (10 \\ Hunan Polytechnic of Environmental and Biology, Hengyang, Hunan 421005, China \\ Correspondence should be addressed to Xiying Deng; dengxiying@hnebp.edu.cn
}

Received 11 November 2021; Revised 16 December 2021; Accepted 6 January 2022; Published 25 January 2022

Academic Editor: Gengxin Sun

Copyright (c 2022 Xiying Deng. This is an open access article distributed under the Creative Commons Attribution License, which permits unrestricted use, distribution, and reproduction in any medium, provided the original work is properly cited.

\begin{abstract}
The fuzzy qualitative approach used in this paper provides an in-depth study and analysis of the simulation of the mental health state of college students, which is continued from another perspective using structural equation modeling, in which sufficient conditions for the equation to satisfy local asymptotic stability, instability, and global attraction at the zero equilibrium point and sufficient conditions for the existence and instability of nonzero equilibrium points are discussed, respectively. In addition, the paper proves the boundedness of the solutions of the equation by mathematical induction and obtains sufficient conditions to ensure that the solutions are bounded. The intrinsic causes of college students' psychological problems lie in the lack of social support in the family and outside the family and the influence of the overall social environment on college students. Finally, this paper puts forward some suggestions and countermeasures based on this research finding from three levels: family, school, and society, giving full play to the role of family support; schools should expand the main body of support, enrich the form of support, and provide comprehensive and multifaceted social support for college students. Finally, the simulation of the equation is carried out to verify the correctness of its conclusion. The rate of mental subhealth is 1.605 times that of college students admitted to ideal universities, which is consistent with our actual situation. At the level of condition guarantee, the hardware facilities, management system, and professional team of mental health education for college students are evaluated; from the specific content, the teaching work, educational activities, psychological counseling, and crisis intervention of mental health education for college students are evaluated; from the perspective of effect, the degree of goal achievement, social adaptation, and result satisfaction of the effect of mental health education for college students are evaluated. The final part analyzes and elaborates on the construction of evaluation indexes of mental health education for college students, the design of weight of guiding indexes, and the content and optimization path of the evaluation test. The results of the study show that personal ability and external pressure are important factors influencing college students' mental health, and the influence of external pressure is greater. Logistic regression and structural equation modeling methods complement each other, and the findings of structural equation modeling are more comprehensive and integrated, while logistic regression illustrates the degree of each influencing factor.
\end{abstract}

\section{Introduction}

It is a method of studying the whole system based on the systemic nature of the object itself. The evaluation of mental health education of new-age college students is a huge but closely connected whole, and when exploring the relevant contents, we should pay attention to the evaluation's wholeness and systemic nature. The evaluation of mental health education of new-age college students should be regarded as a system, not to overemphasize the importance of a certain part, but to consider all the elements in the evaluation system that affect and interact with each other, so that the organic connection between each element can produce an integrated effect. Considering evaluation research as a dynamic whole, it can be adjusted and improved according to the requirements of the times to realize the sustainable development of the evaluation of mental health education of college students in the new era.

As the theory related to difference equations continues to evolve, scholars have encountered two major difficulties, the first being that the models describing the problem are too complex; as the complexity of the system model increases, 
one's ability to accurately depict the relevant properties of the model decreases, so that models built for real-world problems have difficulty in perfectly presenting the nature of the problem [1-4]. The second difficulty relates to the uncertainty caused by the inability to accurately describe the actual situation. Many subjective and objective aspects of the real world cause the known information needed to build and solve difference equation models for many phenomena and things to be incomplete, and the parameters, as well as the initial values in the models, are fuzzy, and to solve this problem, scholars have introduced fuzzy sets in differential equation models. The interpretation of structural equation model analysis results can be divided into two parts, one is the interpretation of structural model results, and the other is the interpretation of measurement model results. The structural model reflects the relationship between the external latent variable and the internal latent variable, and the path coefficient is the regression coefficient; the measurement model reflects the relationship between the external latent variable and its observed variable, and the path coefficient is the factor load of each observed variable on the independent variable.

However, due to the semimature state of college students, they are not ready to cope with the complex and changing social environment, so they are more likely to have psychological pressure, which leads to mental health problems. The employment challenges are not only academic but also postgraduation. In addition, emotional, interpersonal, and economic problems are also problems that college students will face at any time in their study and life and with so many problems and challenges. Once mental health problems occur, not only will it affect the study and life of college students, but also serious mental problems will make college students have physical health problems and even go to extremes. When mental health problems occur, not all college students receive material or emotional support and comfort, and some of them do not receive timely and effective support from the outside world.

\section{Related Work}

The mental health of college students has always been the focus of social attention, and the fields of sociology, psychology, and education have conducted multilevel and allaround research on this topic. Foreign research on this topic started earlier, and with the development of the times, domestic research on this topic has been gradually enriched. The factors influencing the mental health of college students are divided into two aspects, which are endogenous and exogenous factors [5]. The internal factors include physiological factors as well as life habits, and the external factors include family as well as education patterns. Similarly, the factors influencing the mental health of college students are divided into two major aspects, intrinsic and extrinsic, with intrinsic factors including genetic, physiological, and psychological factors and extrinsic factors including family, school, and social factors [6]. Many studies have used the Symptom Self-Rating Scale SCL-90 to test the mental health of college students and questionnaires to collect data and explore the factors that affect college students' mental health. Some researchers used $t$-tests to find that ethnicity, family residence, poverty, regular participation in sports, and ideal school were significantly different in the realization of college student's mental health status and then conducted binary logistic regressions on these factors [7]. There is generally only one top level, which refers to the goal that the research object wants to achieve, which is also the target level; the middle level contains all the relationships from the bottom to the highest level and plays a role in explaining the internal structure, also called the criterion level. The bottom layer contains the methods and measures to achieve the goal, also known as the program layer [8]. However, its school health education model is very worthy of reference.

School health education in the United States and Japan has been borrowed and recognized by many countries, and the field of school health education has been the most studied in the United States and Japan, and the policies, models, and contents of foreign school health education are introduced here on behalf of the United States and Japan [9]. Secondly, for higher-order nonlinear fuzzy difference equations, compared with the research results of first-order fuzzy difference equations, their theoretical results are still relatively few [10]. It is well known that the dynamical properties of nonlinear ordinary differential equations and related applications have been the hotspot of many scholars' attention and research, and there are numerous results, which become the research scope of fuzzy differential equations when people define the parameters and initial values in ordinary differential equations as fuzzy numbers [11].

The study found that human behavior is controlled by four major factor systems such as people's psychological, physiological, organizational, and environmental factors, and using system engineering methods such as the fishbone diagram analysis method, the influence factor weights of human unsafe behavior were reordered, and the hierarchical nature of unsafe behavior was delineated and it was concluded that training and education, management and discipline, effective incentive mechanisms, and a good working atmosphere are important to control human unsafe behavior methods and approaches [12-14]. When studying the operational behavior of coal mine employees, it found that the psychology and thoughts of people who are difficult to control are the key causal factors, and psychological counseling proposed as an important way to solve these psychological problems.

\section{Fuzzy Qualitative Simulation Analysis of College Student's Mental Health Status}

3.1. Fuzzy Qualitative Method Design for Mental Health Status. Difference equations are a discrete form corresponding to continuous differential equations, so the theory related to difference equations has become a research hotspot for many scholars [15]. The difference equation is even regarded as a more classical mathematical model in computer simulation and experiment. Choose the most widely used and authoritative combination of qualitative analysis 
method, empirical determination method, and Delphi method. The specific content of the experience determination method is to consult professional scholars in the field of mental health education for college students and determine the weight of the evaluation based on their rich work experience.

$$
x_{n}=\frac{A x_{n-1}-x_{n-2}}{B+x_{n+1}} .
$$

With the continuous development of nonlinear difference equation theory, scholars found that the known information needed to describe the difference equation model of many practical problems is incomplete, because the parameters and initial values are fuzzy, so people combine fuzzy sets and difference equations to form fuzzy differential equations. In recent years, fuzzy difference equations have become a hot topic of attention for many scholars, and in the study of fuzzy difference equations, fuzzy difference equations can be transformed into a set of rational difference equations by truncated sets for research, and the theory related to the set of rational difference equations has been further advanced.

$$
x_{n-1}=\frac{x_{n+1}^{2}}{B-y_{n} y_{n+1} y_{n-2}^{2}} .
$$

Measurement models consist of latent and observed variables. For example, when studying a person's personality, personality is an abstract concept that cannot be measured directly, so it is necessary to respond to the latent variable of personality through observed variables. For example, the personality can be measured by investigating the number of parties they attend, the number of friends they have, and whether they have good interpersonal relationships. Structural equation modeling can deal with the relationship between several variables at the same time and can reveal the degree of influence between the variables and is a widely used model in psychology and sociology [16]. Personality is a latent variable, and the number of friends, whether they party regularly, and whether they have good interpersonal relationships are the exogenous variables, also called observed variables.

We can use questionnaires to obtain data on observed variables and thus understand the potential variable of personality. The purpose of such educational activities is to solve the psychological problems that have arisen or will arise among college students. As a newly emerged research direction, the evaluation of college students' mental health education has no enough information in the past, scholars have different research perspectives, and it has been given more connotations in the process of changing and developing with the times, so the academic community has not given a very precise definition so far [17].

$$
\begin{aligned}
P(\lambda) & =\left(\lambda^{m}-\frac{A_{1}}{B_{2}^{2}}\right)\left(\lambda^{m-1}-\frac{A_{2}^{2}}{B^{2}}\right), \\
L_{n} & =\frac{A_{l, a} L_{m+n, \alpha}}{B_{r, \alpha}-C_{r, \alpha} R_{n}^{2}} .
\end{aligned}
$$

This part is a bibliometric analysis of journal papers on the topic of college students' health education, extracting keywords, and performing coword analysis after the standardization of keywords. The health problems faced by college students are becoming increasingly diversified and complicated, and according to the changing trend of the volume, the keywords are analyzed by clustering, and the high-frequency keywords in each cluster can be summarized to obtain the research themes and lay the foundation for the next research. Moreover, the traditional concept generally assumes that women are more emotionally rich and sensitive and can perceive others' difficulties and give support. The final evaluation results are highly uniform. The scientific standard refers to the evaluation standard based on scientific principles and objective regulations. It emphasizes selfcomparison rather than other comparisons. It is a procedural evaluation standard and pays more attention to whether the education process follows the law of student development. The role of men generally gives the impression that they are independent and introverted and may not need to seek help from the outside world, while some boys may feel that they experience some respect from the outside world and thus believe that they receive higher subjective support, but there is a greater chance of more confident and conceited people among boys who tend to refuse help from others, while girls prefer to reveal they have true feelings and seek dependence with family and friends.

$$
\sum_{q=1}^{Q}|R(X(t)-Y(t))| \geq \delta .
$$

Internal collaboration of multirobot systems has always been the most challenging challenge in robotics, especially when the group task cannot be decomposed into independent subtasks. The behavioral gains of a single robot depend on the joint effects with other members, and the success of the overall task execution process is more dependent on the collective execution strategy of the robot population. Internal task collaboration becomes a particular challenge for robotic population systems due to the difficulty of real-time and effective task distribution and resource allocation for distributed, self-organizing individual robots in the system. The difficulty in its resource allocation lies in the unknown and uncertainty of the global state of the system for individual robots, which makes it necessary for each robot to make local decisions based on local cognition, which is difficult to meet the group global rationalization requirements. Effective robot group collaboration should satisfy the ability to significantly reduce the programming complexity of multirobot group behavior, the ability of the robot group to dynamically adapt to a changing environment and achieve autonomous decision making of the robot group system in an internally negotiated manner, as shown in Figure 1.

The Emotional Intelligence Network model is fully applicable to the optimization and control problems of complex power systems due to its distributed and self-organizing characteristics, as well as its capability of local collaborative control and node state self-review. When there is an increase 


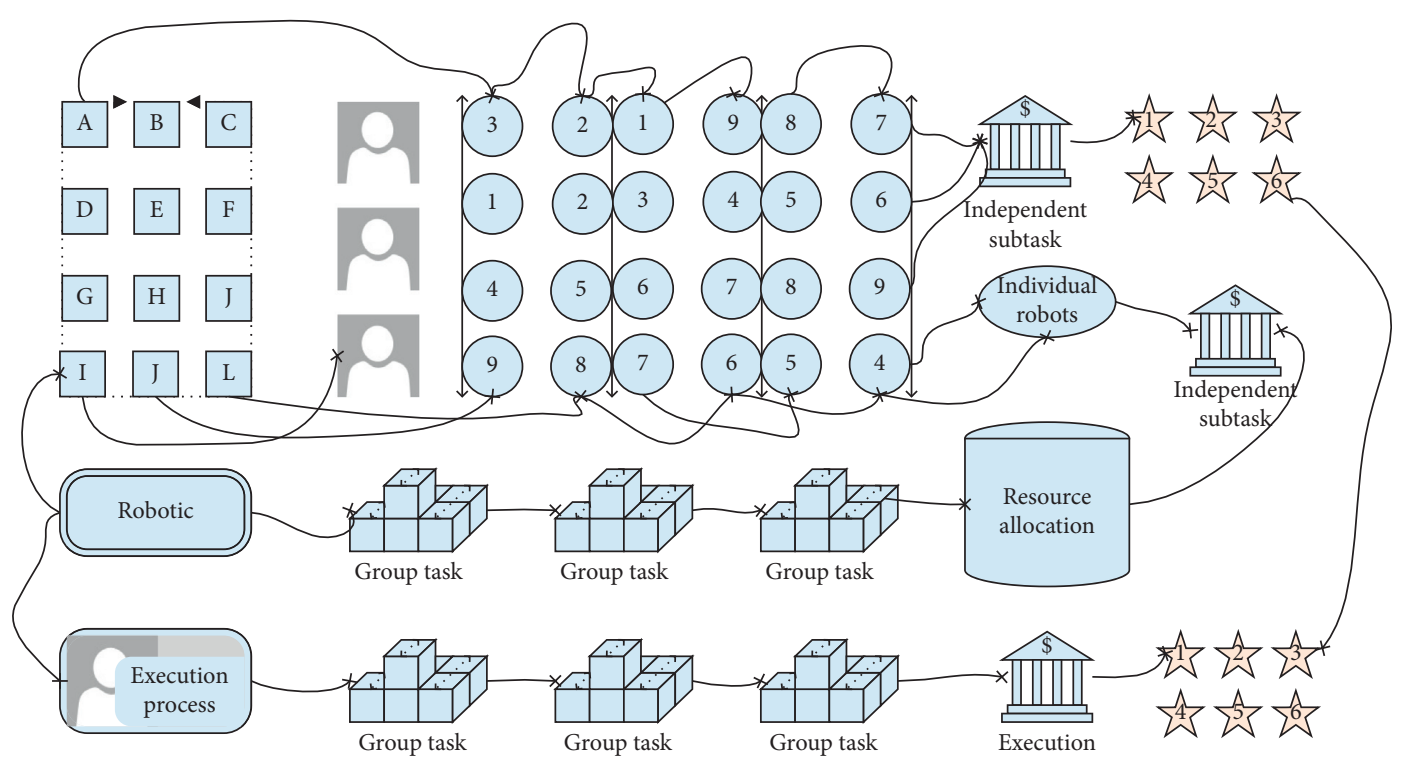

Figure 1: Fuzzy qualitative framework.

of $20 \%$, the number of psychological consultations increased significantly after 14 quarters; when it increased to $30 \%$, the difference between the effect and the effect of an increase of $20 \%$ was not very large, and the rate began to rise in 11 quarters. First, the Emotional Quotient Substation deployed in the Emotional Quotient Network can achieve local power reactive optimization decisions in favor of network-wide optimality based entirely on local data among cooperating power stations without accessing global power information. Second, the Emotional Quotient Substation can provide an accurate emotional representation of its state based on the substation's state data such as power supply, total load, and fault rate for its state assessment and achieve real-time stable feedback control based on the emotional control capability to guarantee the stable operation of the power system.

$$
E\left[|X(t)|^{2}\right] \geq H\left(p^{2}\right) \text {. }
$$

The process of conducting the construction of new counseling indicators mainly includes the qualification of teachers, venue equipment, reception settings, crisis intervention, and developmental counseling effects. The psychological counseling staff should have professional qualifications, the original national second and third level psychological counselor qualifications are the basic access conditions, and the qualifications of the Chinese Psychological Association and other registration systems should be used as the directional requirements for the qualifications of college counselors [18]. As for the reception setting, the new evaluation system will make index construction on whether the existing counseling system can provide reasonable duty, appointment, referral, and desk account.

$$
E\left|\int_{t 1}^{t n} g(t) \mathrm{d} B_{1}(s)\right| \geq\left[\frac{p\left(p^{2}+1\right)}{2}\right]^{p^{2} / 2} N(p) .
$$

Whether the psychological file can objectively record the psychological state of the visiting college students and classify and organize the file information and whether it can strictly abide by the principle of confidentiality, protect students' privacy, and firmly prevent information leakage, the effectiveness of crisis intervention and developmental counseling focuses on whether the counselor can combine psychological problems with actual problems in the counseling process, analyze the different problems faced by students of different majors and sections in a targeted manner, communicate with students more often, truly understand what students think inside, accurately identify the root causes of problems, and then find countermeasures to help students get out of their difficulties. There are also serious mental illnesses and crisis cases that can effectively intervene already with the effectiveness of the intervention. Of course, traditional mental health education tends to take the suicide rate as a very important indicator of the effectiveness of mental health education in colleges and universities. However, due to the complexity of suicide completion factors, it is not objective or scientific to simply attribute it to the quality of mental health education practices or counseling, as shown in Figure 2.

Both stable and cautious personality models produce happy emotions within a short period of time after stimulation, and they fade quickly. The above experimental results show that the four personality models we define can be based on different personality preferences. The scientificity and operability should be taken into consideration when decomposing the three-level indicators. From the dimension of time, the target achievement can be divided into the target achievement indicator of immediate effect and the target achievement indicator of long-term effect. The indicator setting of the goal achievement of immediate effect can quickly understand the achievement of goals and give feedback, to adjust the improper development direction of college students' mental health education based on the present moment, thus gathering sand into a tower. To set indicators for the achievement of long-term goals, it is 


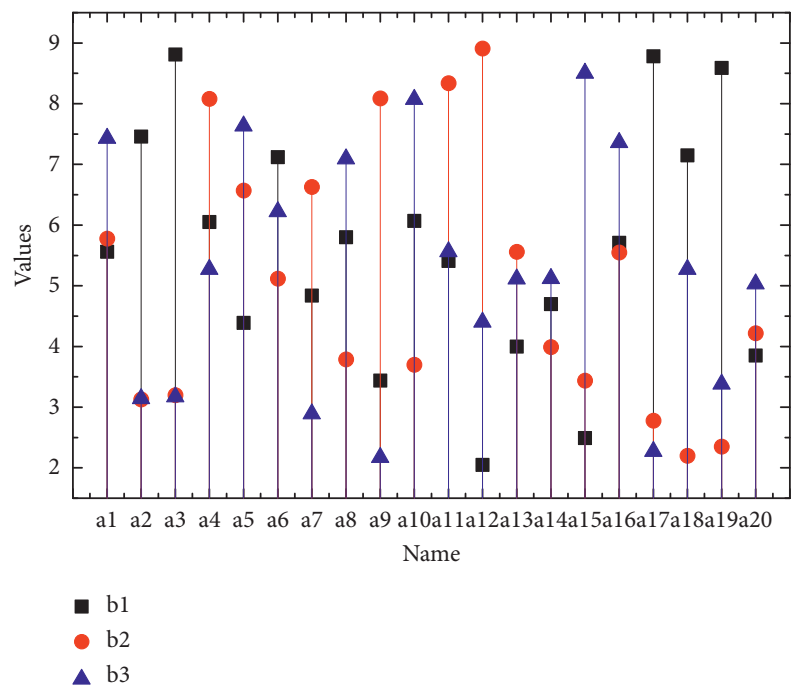

FIGURE 2: Simulation parameters of the stochastic system emotional pulse model.

indispensable to conduct stage evaluation. It is a process to evaluate the achievement of long-term effects, and it is necessary to focus on each stage to do a good job of achieving the overall goal of mental health education for college students. The evaluation of the achievement of the goals of immediate- and long-term effects will coordinate and promote the quality of mental health education for college students.

\subsection{Simulation Design of College Student's Mental Health} Status. This is also consistent with the results of existing studies and suggests that social support plays a significant role in promoting mental and physical health, either directly or indirectly by buffering the damage of stress, as shown in Table 1.

In real life, not every college student can get into the university of their choice. It is important to note that our conclusions are somewhat one-sided due to the small amount of data. For the demographic characteristics that failed to pass the test in this study, we cannot hastily deny their health status, and further research is needed to verify their role. Next, we conducted a multifactor analysis using logistic regression to further explore how demographic factors affect the mental health status. From the regression equation, we know that college students who did not enter their ideal university had a somewhat worse mental health compared to those who entered their ideal university, and their rate of developing mental subhealth was 1.605 times higher than that of college students who entered their ideal university, which is consistent with our actual situation. We believe that college students' mental health is influenced by more demographic characteristics, and we suspect that this result is caused by the small amount of data and that the difference failed to reach statistical significance due to the small amount of data. It is imperative to conduct research on the quality of college students' mental health education. Among them, the evaluation of college students' mental
TABLE 1: Differences in social support across mental health symptom groups.

\begin{tabular}{lccc}
\hline Group & $M$ & SD & $T$ \\
\hline Low symptom group & 29.6 & 2.705 & 27.2 \\
High symptom group & 59.3 & 3.28 & 38 \\
Low symptom group & 89.3 & 3.27 & 30.13333333 \\
High symptom group & 77.2 & 2.18 & 26.66666667 \\
Low symptom group & 33.9 & 3.41 & 28.8 \\
High symptom group & 71.4 & 1.255 & 34.33333333 \\
Low symptom group & 61.6 & 2.235 & 42.06666667 \\
\hline
\end{tabular}

health education is an effective means to improve the quality of education, and it is most worth exploring.

Most of the problems can be solved through counseling, but the problem is that the utilization rate of counseling rooms is very low, and insufficient understanding of counseling leads to students not choosing to solve their psychological problems through counseling, so it is necessary to make students understand counseling correctly; human self-awareness will be gradually improved with growth and will last until middle age. Whether college students can correctly understand themselves and objectively evaluate themselves is crucial to the development of their personality. The student stage is an important period for the development of values, and in today's highly developed information, the factors that affect the formation of college students' values or even change them are complex, and correct values education is an important element of health education.

According to the findings of the study, we can provide students in a targeted manner $[19,20]$. On the one hand, we should give college students course guidance about future planning to help them face up to themselves. On the other hand, it is also crucial for students themselves to enhance their frustration ability and improve their overall quality through continuous exercise. In this section, we try to use the 18 questions in the self-designed questionnaire on living habits directly as independent variables for regression analysis, and the results show that two questions enter the regression equation. We believe that the factors influencing the mental health status of college students are supposed to be more complex, as shown in Figure 3.

Model fitting is the estimation of model parameters. The model needs to be evaluated after fitting the model, and in structural equation models, statistics can be used to test the excellence of the model and data fit. The path coefficients of the exogenous potential variable of personality and the endogenous potential variable failed the test and it was not advisable to remove the variables hastily, so the effect of covariance between the exogenous potential variables was considered. In addition, the individual questions in the questionnaire directly affect the endogenous potential variables. It is also a key and effective way to improve the quality of college students' mental health education. The correlation between the error terms is added based on the correction indicators provided by AMOS, and the reason for this correlation is the presence of variance in the model that is not explained by the theoretical model, which may stem 


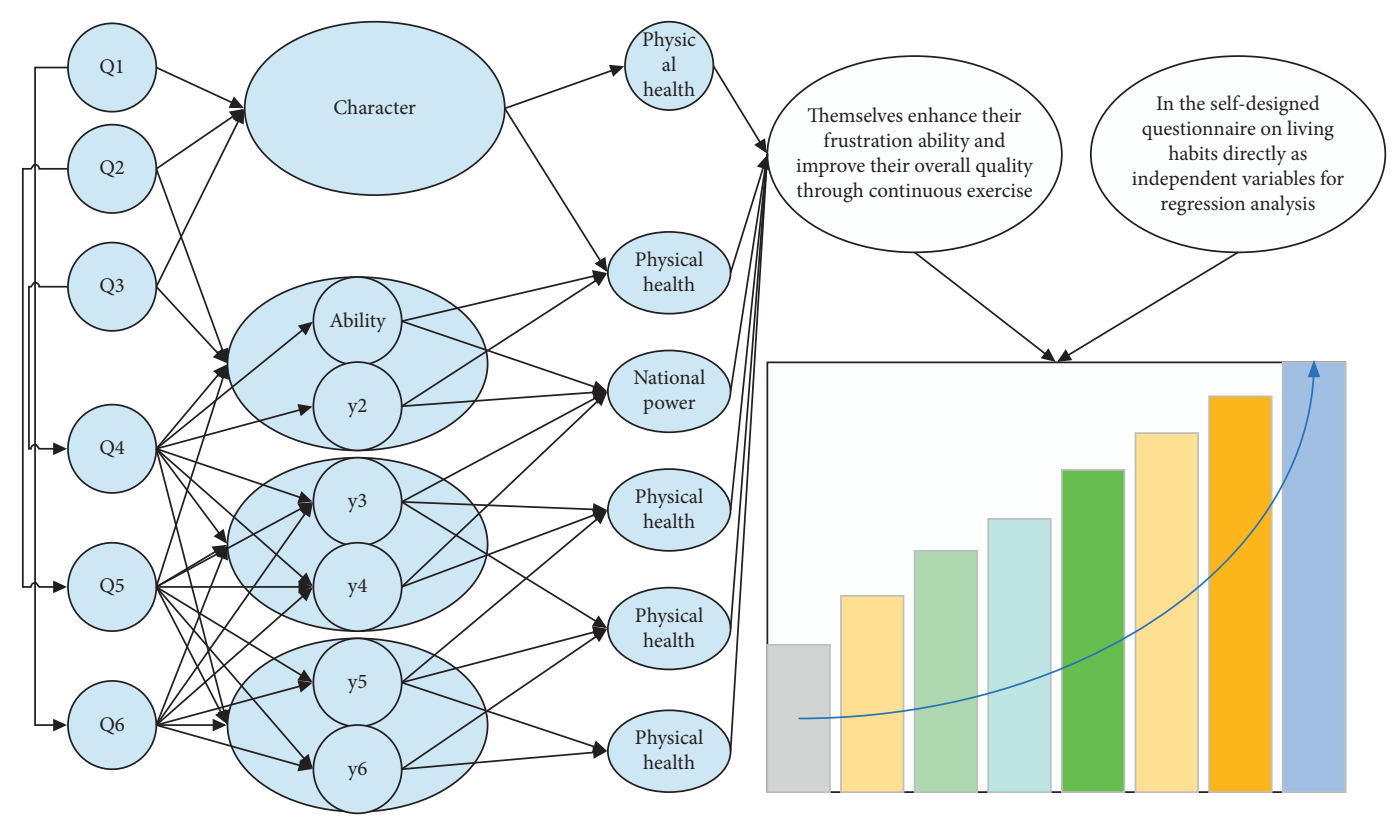

FIgURE 3: Initial model diagram.

from systematic errors in the measurement instruments. In future research, we will continue to study this part in-depth and try to minimize the systematic errors of the measurement tools. The structural model responds to the relationship between the exogenous potential variables and the endogenous potential variables, and the path coefficients are the regression coefficients, and the path coefficients are the factor loadings of each observed variable on the independent variables.

From the final model standardized path coefficients as well as standardized factor loadings for both tables, both potential variables, stress and competence, have a significant effect on mental health, but there are differences between the two, with stress having a somewhat greater effect on mental health, which is in line with our reality. Nowadays, with the development of the economy, education has progressed and there are increased college students, which brings challenges and competition to contemporary college students. In addition, the everevolving technology has made the threshold of employment higher and higher, putting college students under a lot of pressure. In addition to employment, academics, interpersonal relationships, and family are also environments that college students need to face. College students are at a stage where their identity is more complex, and their insufficient psychological maturity combined with the fast pace of life makes them psychologically burdened and inevitably anxious and depressed.

\section{Analysis of Results}

4.1. State Fuzzy Characterization Results. To verify the theoretical results obtained earlier, examples are given here to verify the periodicity, and boundedness, of the solutions of this class of maximal type fuzzy differential equations. In the first step, the object of study is organized and hierarchical. In the model, the problem is decomposed into different components, which are called elements. The general model can be divided into three layers: the top layer, the middle layer, and the bottom layer. It is necessary to adhere to the fundamental task of establishing morality, conform to the development of the times, stimulate the vitality of the times, and carry out the research work on the evaluation of mental health education for college students. The highest level, which is generally only one, refers to the goal that the object of study wants to achieve, also known as the goal level; the middle level contains all the relationships from the bottom level to the top level and explains the internal structure, also known as the criterion level; the bottom level contains the methods and measures to achieve the goal, also known as the solution level. The recursive hierarchy does not limit the number of levels, but to avoid the difficulty of judgment caused by too many elements, the number of elements in each level does not exceed 9, as shown in Figure 4.

After selecting the evaluation indicators of mental students, it is necessary to define the weights of each level of indicators. The function of defining the weight of indicators is to highlight the importance of different indicators in the evaluation system of mental health education of college students, and by determining the weight to show the importance of evaluation indicators at all levels, the evaluator and the evaluated can understand briefly, and the educational problems can be pointed out briefly, to improve mental health education in a targeted manner. The parameters and initial values in the model are fuzzy. To solve this problem, scholars have introduced fuzzy sets in the difference equation model. Although some results have been achieved in the research of fuzzy difference equations, there is still a lot of research space. For example, the weight of indicators such as the guidance of online teaching on 


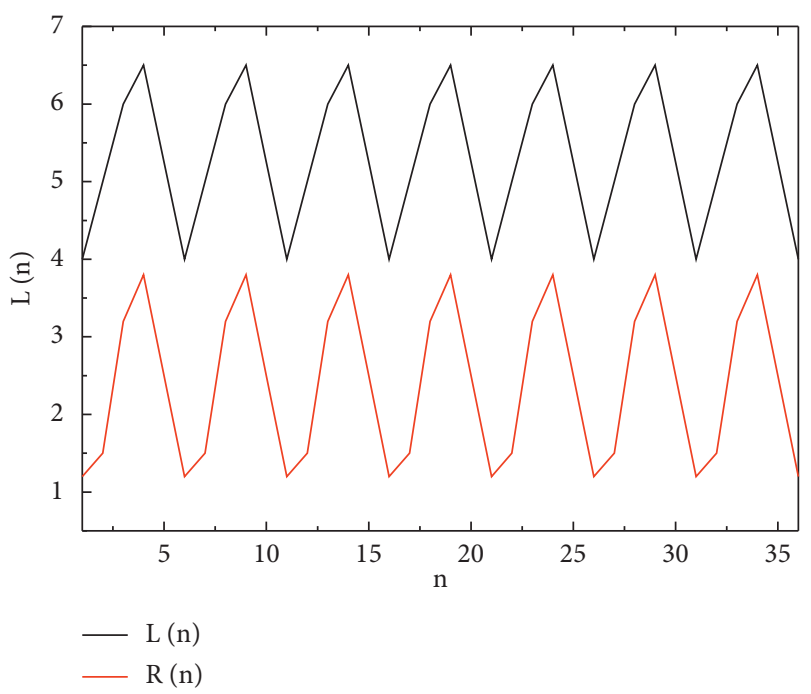

Figure 4: Periodicity of the solution.

students' morality and the value-led role of mental health education activities in the process of mental health teaching will need to be strengthened, to update the evaluation weights and better meet the requirements of the new era.

In the process of defining the weights of specific college student mental health education evaluation indicators, considering the diversity of methods available, the most widely used and authoritative qualitative analysis method, the empirical determination method, and the Delphi method are combined based on the professional characteristics of college student mental health education. The specific content of the empirical determination method is to consult with professional scholars in the field of mental health education for college students and determine the evaluation weights based on their rich working experience. The criteria of indicator evaluation are the yardstick to visually reflect the results of mental health education, as shown in Figure 5. With so many problems and challenges, the mental health of college students is worrying. Once a mental health problem occurs, not only will it affect the study and life of college students, but serious psychological problems will cause college students to have physical health problems or even go to extremes.

There exist many classifications of evaluation standards; for example, from the evaluation content, it can be divided into quality standards, duty standards, and effectiveness standards or into state standards and effect standards; from the reference standards, it can be divided into social standards and scientific standards. It is reasonable to set the threshold of evaluation standards based on social standards and scientific standards. Social standards refer to the evaluation standards based on the unified requirements of documents, policies, and regulations issued by the national education department, and the final evaluation results are highly uniform. The scientific standard refers to the evaluation standard based on scientific principles and objective regulations, which emphasizes self-comparison rather than another

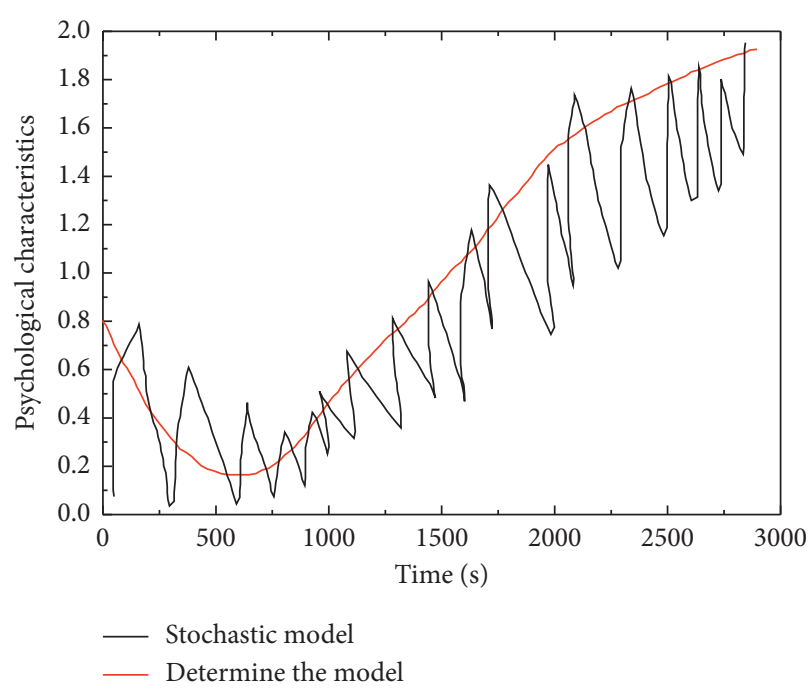

Figure 5: Psychological certainty versus randomness model.

comparison, and is a kind of process evaluation standard that pays more attention to whether the education process follows the law of students' development and avoids only taking the evaluation result as the only judgment standard, which is more in line with the new era of humanoriented education evaluation concept. Due to the special nature and complexity of mental health education, a single evaluation standard is not able to meet the complex and huge evaluation system, and personalized evaluation standards should also be applied and adopted.

\subsection{Simulation Results of College Student's Mental Health} Status. At present, the content of any college student mental health education evaluation system cannot guarantee absolute comprehensiveness and ensure that the indicators of the education evaluation system perfectly contain all the details, which requires the sustainable development of the evaluation system construction and ensures the timely updating of the innovative content of college student mental health education evaluation and renewing the vitality of the times. It is undeniable that optimizing the evaluation content is a long and continuous journey, and future development is a long way to go. The development of modern society is dynamic to the requirements of higher education, and the content of educational evaluation cannot remain unchanged; not only will there be additions to the content, but also the existing system contains content that may no longer meet the needs of the times and needs to be modified or deleted. The evaluation of university students' mental health education should be in line with the political, economic, and cultural development and should also adapt to the requirements of ideology on students' psychological quality in different periods, as shown in Figure 6. Personality is a latent variable. The number of friends, whether they often go to parties, and whether they have good interpersonal relationships are their explicit variables, which are also called observed variables. 


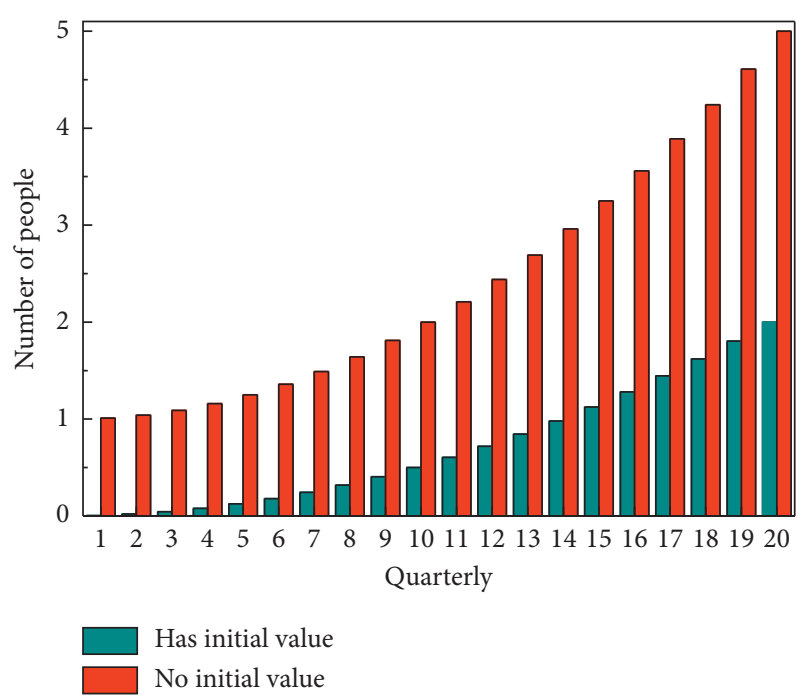

FIgURE 6: Counseling behavior simulation diagram.

The whole curve is from showing a slow decline, with a slowly decreasing rate of decline, to a turnaround at quarter 13 , when the decline turns to an increase and an increasing rate of increase. Internal task collaboration has become a special challenge for robot swarm systems. The difficulty of its resource allocation lies in the unknown and uncertainty of the global state of the system to individual robots, so that each robot must make local decisions based on local cognition, which is difficult to meet the group's global rationalization requirements. At this stage of the business, without these measures, there is not much impact on the number of counseling, but the number of people has indeed been in a state of decline; these measures are a subtle influence on people's behavior; the effect is not obvious at first, but after the accumulation of time, the demand for counseling is increasing and translates into an increase in the rate of counseling behavior not considered to increase. From the comparison of the curves with and without initial values, it is concluded that companies that have had similar strategies are more likely to increase the number of people in psychological counseling and the gap widens over time.

The graphs reflect that when the total intervention is increased by $10 \%$, it is almost the same as the initial state, so an increase in the intensity of only $10 \%$ will not have a great effect; when it is increased by $20 \%$, the number of psychological counseling is significantly enhanced after 14 quarters; when it is increased to $30 \%$, the difference between the effect and the effect of increasing by $20 \%$ is not very large, and the rate starts to rise at 11 quarters. So, when you want to increase the overall strategy on the number of psychological counseling, it is better to be above $10 \%$ and as far as possible to reach about $20 \%$, and if time and various conditions are more adequate, you can consider raising to $30 \%$, thus reducing the number of declines. So, a reasonable intensity increase would be around $20 \%$ on top of the original, as shown in Figure 7.

The dominant personality model produced a pleasurable emotion shortly after the stimulus ended, and this

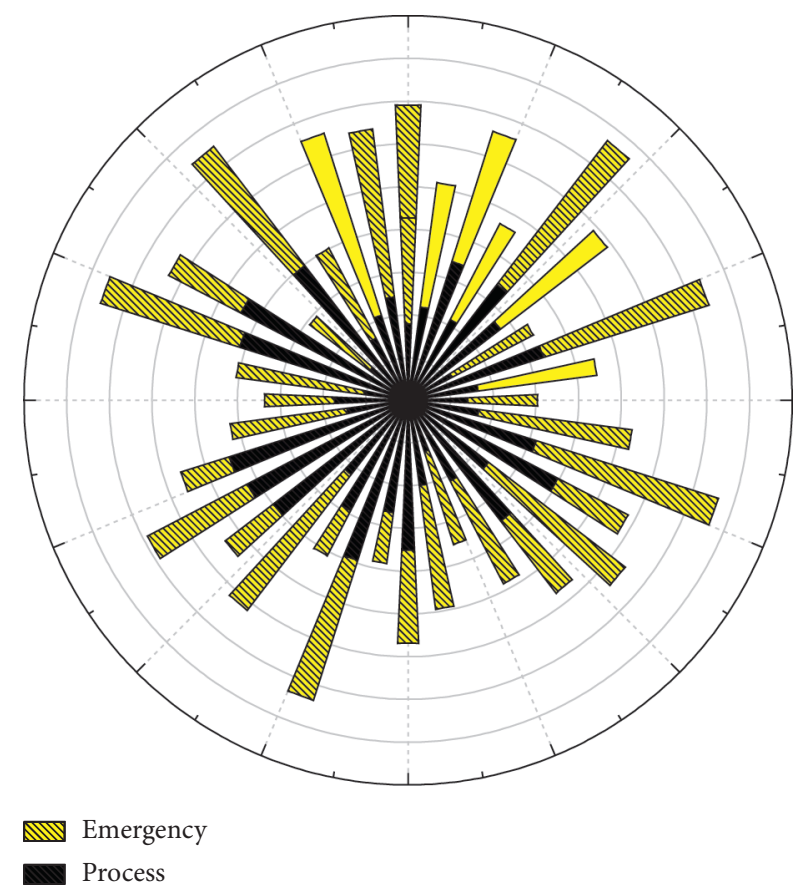

FIGURE 7: Output arrival time of mental state identification results.

emotion was quickly restored. The effective personality model was able to respond immediately to the pleasurable stimulus and continued to produce pleasurable emotions during the stimulus. Both the steady and cautious personality models produced happy emotions for a short time after the stimulus and quickly faded. The above experimental results suggest that the four personality models we defined can produce different emotional experiences with the same stimuli depending on different personality preferences. We can notice that the response output of the system always lags slightly behind the stimulus input, with an average time difference of about $0.03 \mathrm{~s}$, which indicates that the intracranial emotion takes some time to compute.

\section{Conclusion}

The evaluation of mental health education of university students has an irreplaceable role in the improvement of the quality of education, and the evaluation of mental health education of university students allows showing more visually the progress of a certain educational stage and its problems. Evaluation of quality is a new kind of evaluation that goes beyond quantitative evaluation and is a higher type and level of evaluation activity. Communicate with students more, truly understand what college students are thinking, accurately and accurately find out the root cause of the problem, and then find countermeasures to help students get out of the predicament. There are also cases of severe mental illness and crisis that can effectively intervene the effectiveness of the intervention. In the context of the new era, the introduction of the concept of moral education has pointed out the direction for college mental health education, and the value judgment and development direction of its quality has 
been further defined. Mental health education evaluation of college students is currently a relatively weak part of the mental health education work system in colleges and universities, but at the same time, it is also a key way and effective way to improve the quality of mental health education of college students. Therefore, it is of certain practical significance for the thesis to update and improve the evaluation system of college students' mental health education according to the needs of the contemporary background. In a word, if we want to realize the mission of the times to improve the quality of mental health education of college students and then improve the quality of ideological and political education in colleges and universities, we should adhere to the fundamental task of establishing moral education, conform to the development of the times, stimulate the vitality of the times, and carry out the research work on the evaluation of mental health education of college students strongly. Building a good and complete social support system requires enriching the members of the social support network of college students, integrating the resources of the social support network, and playing the functions of the social support network. Only by establishing such a social support system can we prevent, treat, and maintain the mental health problems of college students comprehensively, with the support of public policies provided by the government and the participation and joint efforts of the whole society, such as families, schools, communities, social organizations, and the government.

\section{Data Availability}

The data used to support the findings of this study are available from the author upon request.

\section{Conflicts of Interest}

The author declares no conflicts of interest.

\section{Acknowledgments}

This work was supported by the project of Excellent Team Building Project of Hunan University Excellent Ideological and Political Workers Project in 2021 (no. 26).

\section{References}

[1] J. Wang, Z. Zhang, H. Luo, Y. Liu, W. Chen, and G. Wei, "Research on early warning model of college students' psychological crisis based on genetic BP neural network," American Journal of Applied Psychology, vol. 8, no. 6, pp. 112-120, 2019.

[2] A. Ströhle, "Sports psychiatry: mental health and mental disorders in athletes and exercise treatment of mental disorders," European Archives of Psychiatry and Clinical Neuroscience, vol. 269, no. 5, pp. 485-498, 2019.

[3] Q. Liu, Y. Wang, M. Z. Yao, Q. Tang, and Y. Yang, "The effects of viewing an uplifting 360-degree video on emotional wellbeing among elderly adults and college students under immersive virtual reality and smartphone conditions,"
Cyberpsychology, Behavior, and Social Networking, vol. 23, no. 3, pp. 157-164, 2020.

[4] A. B. R. Shatte, D. M. Hutchinson, and S. J. Teague, "Machine learning in mental health: a scoping review of methods and applications," Psychological Medicine, vol. 49, no. 9, pp. 1426-1448, 2019.

[5] M. A. Piot, A. Dechartres, C. Attoe et al., "Simulation in psychiatry for medical doctors: a systematic review and meta-analysis," Medical Education, vol. 54, no. 8, pp. 696-708, 2020.

[6] A. S. Gabriel, J. T. Campbell, E. Djurdjevic, R. E. Johnson, and C. C. Rosen, "Fuzzy profiles," Organizational Research Methods, vol. 21, no. 4, pp. 877-904, 2018.

[7] A. Pilkington, P. Bowen, R. C. Rose, D. R. Rajasinghe, and I. Evans, "A qualitative study of student retention: the University Academic's perspective," International Journal of Academic Multidisciplinary Research, vol. 2, no. 10, pp. 19-28, 2018.

[8] E. Shahrestanaki, K. Maajani, M. Safarpour, H. H. Ghahremanlou, M. Sahebkar, and A. Tiyuri, "The relationship between smartphone addiction and quality of life among students at Tehran University of medical sciences," Addicta: The Turkish Journal on Addictions, vol. 7, no. 1, pp. 61-66, 2020.

[9] C. J. Beemer, K. A. Stearns-Yoder, S. J. Schuldt et al., "A brief review on the mental health for select elements of the built environment," Indoor and Built Environment, vol. 30, no. 2, pp. 152-165, 2021.

[10] Y. Zheng, Q. Kang, J. Huang et al., "The classification of eating disorders in China: a categorical model or a dimensional model," International Journal of Eating Disorders, vol. 52, no. 6, pp. 712-720, 2019.

[11] Y. Mekawi, C. B. Lewis, N. N. Watson-Singleton et al., "Racial identity profiles among suicidal black women: a replication and extension study," Journal of Black Studies, vol. 51, no. 7, pp. 685-704, 2020.

[12] C. W. Rudolph, D. P. Costanza, C. Wright, and H. Zacher, "Cross-temporal meta-analysis: a conceptual and empirical critique," Journal of Business and Psychology, vol. 35, no. 6, pp. 733-750, 2020.

[13] M. Priscilla Dooshima, E. N. Chidozie, B. J. Ademola, O. Olusanya, and P. A. Idowu, "A predictive model for the risk of mental illness in Nigeria using data mining," International Journal of Immunology, vol. 6, no. 1, pp. 5-16, 2018.

[14] G. Schroers, J. G. Ross, and H. Moriarty, "Nurses' perceived causes of medication administration errors: a qualitative systematic review," Joint Commission Journal on Quality and Patient Safety, vol. 47, no. 1, pp. 38-53, 2021.

[15] J. G. Kheswa, M. N. Mahola, and X. Dayi, "The effects of cultural practices and substance abuse among male students at one South African College Campus," Journal of Human Ecology, vol. 70, no. 1-3, pp. 58-66, 2020.

[16] K. Memmedova, "Quantitative analysis of effect of Pilates exercises on psychological variables and academic achievement using fuzzy logic," Quality and Quantity, vol. 52, no. 1, pp. 195-204, 2018.

[17] D. Luta, E. Pogrebtsova, and Y. Provencher, "The wellbeing implications of thinking about schoolwork during leisure time: a qualitative analysis of Canadian university students' psychological detachment experiences," Journal of Further and Higher Education, vol. 45, no. 6, pp. 771787, 2021.

[18] I. Han, "Immersive virtual field trips in education: a mixedmethods study on elementary students' presence and 
perceived learning," British Journal of Educational Technology, vol. 51, no. 2, pp. 420-435, 2020.

[19] C. Yue and X. Xu, "Review of quantitative methods used in Chinese educational research, 1978-2018," ECNU Review of Education, vol. 2, no. 4, pp. 515-543, 2019.

[20] F. A. Bourini, N. M. Aljawarneh, I. F. Bourini, M. F. Almaaitah, and K. A. K. Almori, "Directing strategic decision and perceived faculty performance using PLS analysis and Monte Carlo simulation in Jordanian private universities," Journal of Talent Development and Excellence, vol. 12, no. 3s, pp. 2235-2252, 2020. 